\title{
Exploring specificity of speeded aiming movements: Examining different measures of transfer
}

\author{
ERICA L. WOHLDMANN \\ California State University, Northridge, California \\ AND \\ Alice F. Healy \\ University of Colorado, Boulder, Colorado
}

\begin{abstract}
Participants were trained and tested to move a mouse cursor from a start position to targets on a circular display in a perceptual-motor reversal condition, with horizontal, but not vertical, reversals. During training, some participants (experimental) moved to two targets either along a single diagonal axis (D1) or along both axes (D2). For D2, return movements from the targets were in the same direction as instructed movements to unpracticed targets. Others (control) trained on all targets. Testing always involved all targets. At test, movement times (to reach the target after leaving the start position) were shorter on trained than on untrained targets, especially for the D1 condition, documenting training specificity. However, movement times in the experimental conditions to new targets during testing were shorter than those in the control condition during training, documenting transfer of learning, with more transfer for D2 than for D1. Initiation times (to leave the start position after target onset) showed no transfer. The results provide evidence that specificity and transfer are not mutually exclusive and depend on the measure used to assess performance.
\end{abstract}

Researchers interested in understanding the conditions that promote skill transfer have often demonstrated that learners lack the ability to generalize beyond the situations presented during training. These findings are consistent with various theoretical accounts, including those involving transfer-appropriate processing (Morris, Bransford, \& Franks, 1977; Roediger, Weldon, \& Challis, 1989), encoding specificity (Tulving \& Thomson, 1973), procedural reinstatement (Healy, Wohldmann, \& Bourne, 2005), and specificity of practice in motor learning (Proteau, Marteniuk, \& Lévesque, 1992). One purpose of the present study is to further explore the conditions demonstrating specificity of learning and those promoting generalizability of learning.

There are ways to enhance transfer of knowledge, and one such way is by introducing sources of variability in practice (e.g., Schmidt \& Bjork, 1992; Wulf \& Schmidt, 1997). However, the advantages of training variability appear to be limited to certain conditions, and these conditions are not well understood. For example, in a previous study (Healy, Wohldmann, Sutton, \& Bourne, 2006), we found no advantage for variability of practice. Specifically, participants learned to perform a speeded aiming task, which involved finding on the display and moving a mouse cursor from a center start position to target digits that appeared along the circumference of a circular stimu- lus array (see Figure 1). During training, the participants practiced various types of perceptual-motor reversals, including a horizontal reversal (in which movements of the mouse to the left resulted in cursor movements to the right, and vice versa, but vertical movements remained intact), a vertical reversal (in which vertical movements were reversed but horizontal movements remained intact), and both types of reversals in one condition. Practice on one reversal resulted in improved performance on that reversal during training, and learning persisted across a 1-week retention interval. However, the learning was highly specific and did not transfer to the use of a mouse with a different type of reversal. In addition, training with a variety of reversals did not result in more transfer than training with only the single reversal included during testing. Thus, the findings did not support the variability-of-practice hypothesis but, instead, were explained in terms of a global inhibition strategy, according to which all normal mouse movements are inhibited when participants encounter a reversed mouse. However, for a mouse with a reversal along only one dimension, participants must disinhibit movements in order to reach the targets along a nonreversed dimension. Because the disinhibition requires an additional step in processing, applying the global inhibition strategy is predicted to result in longer movement times to targets along the nonreversed dimension than to those along the 


\section{8}

\section{7 \\ 1 \\ 6 \\ ห \\ 3 \\ 4}

Figure 1. Circular stimulus display. The target in the display shown is 1 .

reversed dimension - a pattern of movement times that was found by Healy et al. (2006).

Unlike in the study by Healy et al. (2006), in a more recent study (Wohldmann, Healy, \& Bourne, 2008), we did find evidence to support the variability-of-practice hypothesis. Specifically, in Experiment 1, participants were trained and tested using a mouse that was reversed horizontally but not vertically. During training, the participants practiced moving to targets located on either the horizontal (Targets 2 and 6) or vertical (Targets 4 and 8 ) dimension as well as on one of two diagonal dimensions (Targets 1 and 5 or 3 and 7). During testing, the participants practiced moving to all eight targets. In Experiment 2, participants were also trained and tested using the horizontal mouse reversal, but training was limited to a single dimension: vertical (Targets 4 and 8), horizontal (Targets 2 and 6), or a diagonal (Targets 1 and 5 or 3 and 7). As in Experiment 1, the participants practiced moving to all targets during testing.

Wohldmann et al. (2008) measured both initiation time (IT) and movement time (MT), two components of total response time that offer different insights into cognitive processes. Specifically, IT - the time that it takes participants to leave the center start position after the onset of the target digit-reflects encoding of targets and planning of movements (see, e.g., Bourne, Healy, Pauli, Parker, \& Birbaumer, 2005; Elliott \& Lee, 1995; Khan, Lawrence, Buckolz, \& Franks, 2006; Rosenbaum, 1980), whereas MT - the time that it takes participants to reach the target digit after they have left the center start position-reflects motor execution. If variability of practice promotes transfer, shorter response times to new targets should have occurred during testing in Experiment 1 , which involved practice with four different target locations, than in Experiment 2, which involved practice with only two different target locations. That result was in fact observed, although it was restricted to MTs for new targets at test. Additional evidence of transfer was also found. That is, MTs to the untrained targets at the start of testing were significantly shorter than those to the same targets when given to other participants at the start of training. This finding was evident both when training involved four targets (in Experiment 1) and when it involved only two targets (in Experiment 2), although it was significant in only some conditions. However, the results from both experiments also demonstrated specificity effects. In particular, ITs and MTs during testing were significantly shorter for trained diagonal targets than for untrained diagonal targets in both Experiment 1 and Experiment 2.

The findings from Healy et al. (2006) and Wohldmann et al. (2008) demonstrate the need to better define the conditions under which variability of practice results in transfer, which is one purpose of the present experiment. That is, only certain types of variability appear to be effective at promoting generalization of learning. One crucial difference between Healy et al. (2006) and Wohldmann et al. (2008) was that different mouse reversals were compared in the first study, with the targets held constant, whereas different targets were compared in the second study, with the mouse reversal held constant. Thus, it could be that variable practice aids test performance, and training in one condition transfers to other conditions, only when the same generalized motor program, or schema (Schmidt, 1975; i.e., in this case, a mouse requiring the same configuration of movement inhibitions and disinhibitions), is used during training and testing.

In both of our previous examinations of transfer (Healy et al., 2006; Wohldmann et al., 2008), only the instructed movements from the start position to a given target were considered, not the required return movements from the target back to the start position, and it is possible that participants treat the two types of movement differently. More specifically, instructed, but not return, movements could be viewed as intentional; therefore, instructed movements could involve intentional learning, whereas return movements could involve incidental learning. Previous researchers have shown that intentional learning is more effective than incidental learning under some circumstances (e.g., Eagle \& Leiter, 1964; Marmie \& Healy, 2004). Thus, in the present experiment, we extended our previous investigations by further exploring the results of training on a subset of targets to determine whether there is transfer of only the instructed movements or also of the return movements made by participants. We also explored the role of intention in learning to aim toward different targets (i.e., to reach in different directions). Specifically, we compared two experimental conditions, both of which involve training on two of the four diagonal targets but testing on all eight targets. In one condition (D1), both trained targets occurred along a single diagonal axis, whereas in the other condition (D2), the trained targets occurred within two different diagonals. In the D2 condition, movements in all four diagonal directions (northeast, northwest, southeast, southwest) would be learned if both instructed and return movements contribute to learning. In 
contrast, in the D1 condition, movements in only two of the four diagonal directions (either northeast and southwest or southeast and northwest) would be learned. As is predicted by the variability-of-practice hypothesis, more transfer should be evident for participants in the D2 condition than for those in the D1 condition. Likewise, because movements in all four diagonal directions are learned in D2 but not in D1, considering both instructed and return movements, specificity of training should be higher in the D1 condition than in the D2 condition. That is, at test, there should be a greater old-new difference with respect to movements instructed during training for D1 than for D2. Differences between the D1 and D2 conditions should also illuminate any differences between the learning of instructed and return movements. If D2 shows shorter response times at test for the movements that had been instructed during training than for those that had served only as return movements during training, there would be evidence that instructed movements are learned to a greater extent than are return movements.

In a control condition (C), participants were trained and tested on all eight targets. This condition serves as a baseline with which to examine transfer under conditions in which testing and training involve the same number of targets, because it is well-known from the Hick-Hyman law (Hick, 1952) that response time increases as the number of targets increases.

Unlike the variability-of-practice hypothesis, which predicts more transfer for the D2 than for the D1 condition, on the basis of the global inhibition hypothesis alone, full transfer (i.e., no difference between trained and untrained movements at test) should be evident for both the D1 condition and the $\mathrm{D} 2$ condition, because the configuration of inhibition and disinhibition is the same in both conditions. In the present study, unlike the earlier studies, we tested variability of practice and global inhibition predictions by considering two types of transfer: partial and full. To assess partial transfer, we compared response times at the beginning of training in the control (C) condition to those at the beginning of testing in the experimental (D1 and D2) conditions. To assess full transfer, we compared response times at the beginning of testing in all three conditions. Because of the improvement in performance from the beginning to the end of training, full transfer is predicted by the global inhibition hypothesis for both D1 and D2, whereas only partial transfer is predicted by the variability-of-practice hypothesis at least for the D1 condition.

In addition to partial and full transfer, we also separately examined both near and far transfer. Because training in the D1 and D2 conditions involved diagonal targets exclusively, near transfer was defined as transfer to new (i.e., untrained) diagonal targets, whereas far transfer was defined as transfer to new targets along the major (horizontal, vertical) axes. According to the global inhibition hypothesis, the two experimental conditions should show full near and far transfer. In contrast, according to the variability-of-practice hypothesis, the D2 condition should show more near and far transfer than the D1 condition, again assuming that both instructed and return movements contribute to learning.

\section{METHOD}

\section{Participants}

Sixty-six undergraduate students from California State University, Northridge, participated for credit in an introductory psychology course. The participants were assigned by fixed rotation depending on time of arrival for testing to one of the three conditions (D1, D2, or C), with 22 participants in each condition, with the exception that the first 36 participants tested were restricted to the two experimental conditions (D1 and D2).

\section{Apparatus, Materials, and Procedure}

The task involved finding and moving to a single target digit by moving the mouse cursor as quickly as possible from a center starting point to the correct location on the circumference of the clock face. The participants sat approximately $0.5 \mathrm{~m}$ in front of an iMac monitor screen (oriented as normal) that displayed a picture consisting of a center starting point (labeled with an " $X$ ") surrounded by a circle of equally spaced digits (in order, 1-8), with the target for a given trial shown just above the starting point (see Figure 1). The center starting point and the target digits were each surrounded by an invisible circle approximately $1.6 \mathrm{~cm}$ in diameter, and the diameter of the clock face display was approximately $20 \mathrm{~cm}$. The monitor screen size was set at its largest value $(640 \times 480$ pixels $)$, so the display filled the entire screen. The computer program for reversing mouse cursor movements was written in Hypercard, and movement trajectories (including entries into incorrect digit locations) were not captured, so kinematic analyses were not possible. Tape was applied to the mouse cord approximately $15 \mathrm{~cm}$ above the top of the mouse so that the participants could still easily use the mouse in its upright position but could not rotate it upside down. All of the participants were monitored carefully throughout the entire experiment.

On each trial, the participants were required to place the cursor on the " $\mathrm{X}$ " starting point, and the target digit appeared. Next, they were to move to the correct location on the clock face, and when they landed there, the circle surrounding the target digit was highlighted in black. Finally, the participants were to move back to the center starting point to conclude the trial and begin the next. A new trial could not begin until the correct location was reached. During training, a brief demonstration of the task requirements was presented as an animation on the computer screen, and the participants were told, "First place the cursor on the ' $\mathrm{X}$ ' in the center of the screen. Next, wait for the target digit to appear above the ' $\mathrm{X}$.' Then move the cursor to the correct target digit. Last, move the cursor back to the ' $\mathrm{X}$ ' in the center of the screen." Verbal instructions emphasized that the participants were to make their movements as rapidly as possible. The participants in the two experimental conditions (D1 and D2) were given only two of the eight targets to practice during this training phase, with eight trials with each target in each block arranged in a pseudorandom order. More specifically, half of the participants in the D1 condition practiced moving along one diagonal axis (between Targets 1 and 5), and the remaining half practiced moving along the opposite diagonal axis (between Targets 3 and 7). Those in the D2 condition practiced moving along both diagonal axes, with half of the participants practicing on targets on the left side of the clock face (Targets 5 and 7) and the remaining half practicing on the right side of the clock face (Targets 1 and 3). The participants in the control condition (C) were given all eight targets with which to practice, with two trials with each target in each block arranged in a pseudorandom order. All of the participants were required to use the horizontally reversed mouse.

Following a 5-min break, during which the participants were asked to rest quietly, testing began. The same animated demonstration and instructions were given to the participants. Next, the participants practiced moving to all target locations, again using the horizontally reversed mouse. Each of the 12 blocks of testing included two trials with each of the eight targets arranged in a pseudorandom order.

During both training and testing, all of the participants were given the trials in the same order. There were 192 experimental trials in 
each phase, divided into 12 blocks of 16 trials. There were an additional 8 extra trials at the end of each phase that were not part of the analyses, because not all targets were included. Finally, to ensure that all learning would occur during the experimental trials, no opportunity for prior practice was given, and the participants were not made aware of the horizontal mouse reversal in advance.

\section{Design}

A $3 \times 2 \times 12$ mixed factorial design was used, including condition (single diagonal, D1; both diagonals, D2; control, C) as a between-subjects variable and phase (training, testing) and block (1-12) as within-subjects variables. The analyses of training, retention, specificity, and near transfer were restricted to response times (MT and IT, separately) to targets along the diagonal axes, sometimes included only 5 of the 12 blocks, and, in some cases, included test target type (old, new) as a factor. The analyses of far transfer were similar to those for near transfer but were restricted to response times to targets along the major axes (horizontal, vertical), as opposed to the diagonals, and included axis as a factor. Movements along these axes had not been trained in the D1 and D2 conditions, in which training was restricted to the diagonals. The dependent variables were MT and IT. Accuracy could not be assessed, given that the participants had to reach the correct target location in order to proceed to the next trial and because, as was previously mentioned, trajectory data were not captured.

\section{RESULTS}

The results for MT and IT are summarized in Figures 2 and 4, respectively, for diagonal targets and in Figures 3 and 5, respectively, for targets along the major axes during both training and testing as a function of condition, block, and target type. All significant $(p<.05)$ effects are reported here. All analyses were limited to targets along one of the two diagonal axes, except in the case of far transfer. In the analyses of retention and transfer, the training times were limited to those involving the control (C) condition, which served as a baseline, because only that condition included the same number of targets at training as were used at test. These analyses necessarily involved betweensubjects, rather than within-subjects, comparisons, so the assessment of retention and transfer is only indirect.

\section{Movement Time}

Training. We examined training on the three conditions by comparing MTs on all 12 blocks as a function of condition. We found that MTs decreased as a function of practice $\left[F(11,693)=62.70, M S_{\mathrm{e}}=0.331, p<.01\right]$. There were no significant effects involving condition.

Retention. To examine retention, we compared response times on the last five blocks of training in the $\mathrm{C}$ condition to those on the first five blocks of testing in the D1 and D2 conditions for old targets. The main effect of condition was significant, with shorter MTs evident during testing in the D1 condition $(M=1.011 \mathrm{sec})$ and the $\mathrm{D} 2$ condition $(M=0.916 \mathrm{sec})$ than during training in the $\mathrm{C}$ condition $(M=1.196 \mathrm{sec})\left[F(2,63)=3.24, M S_{\mathrm{e}}=\right.$ $0.688, p<.05]$. In addition, separate analyses were conducted comparing each experimental condition with the control condition. For D1, the effect of condition was not significant $\left[F(1,42)=2.83, M S_{\mathrm{e}}=0.663, p=.10\right]$, but for D2, the effect of condition was significant $[F(1,42)=$ $\left.6.39, M S_{\mathrm{e}}=0.676, p=.02\right]$, suggesting perfect retention for both experimental conditions across the 5-min delay and improvement for the D2 condition. In addition, the main effect of block was significant in the overall analysis, suggesting that MTs improved across blocks of training and testing $\left[F(4,252)=7.58, M S_{\mathrm{e}}=0.135, p<.01\right]$.

Specificity. Specificity was assessed by comparing differences in response times to old and new targets at test in the two experimental conditions (D1 and D2). If response times to old targets were found to be shorter than those to new targets, there would be some evidence for specificity of training.

An ANOVA was conducted to examine testing along the diagonal axes in the experimental conditions. MTs to old target digits $(M=0.881 \mathrm{sec})$ were significantly shorter than those to new digits $(M=1.125 \mathrm{sec})[F(1,42)=$ $\left.33.25, M S_{\mathrm{e}}=0.472, p<.01\right]$, demonstrating specificity of training. The old-new difference was larger for participants in the D1 condition (old, $M=0.901 \mathrm{sec}$; new, $M=1.263 \mathrm{sec}$ ) than for those in the D2 condition (old, $M=0.862 \mathrm{sec}$; new, $M=0.988 \mathrm{sec})$; the interaction of condition and target type was significant $[F(1,42)=$ $\left.7.84, M S_{\mathrm{e}}=0.472, p<.01\right]$. Planned comparisons revealed that the main effect of target type was significant for both $\mathrm{D} 1\left[F(1,21)=30.21, M S_{\mathrm{e}}=0.573, p<.01\right]$ and $\mathrm{D} 2\left[F(1,21)=5.60, M S_{\mathrm{e}}=0.371, p=.03\right]$. In addition, in the overall analysis, the main effect of block was significant $\left[F(11,462)=16.31, M S_{\mathrm{e}}=0.102, p<.01\right]$, demonstrating general improvement in MTs across blocks of testing (see Figure 2). The interaction of block and target type was not significant, but the interaction of block and condition was significant $\left[F(11,462)=2.28, M S_{\mathrm{e}}=\right.$ $0.102, p=.01]$, reflecting more improvement across test blocks for D1 than for D2.

Near transfer. For the assessment of near transfer, as for the assessment of training and retention, only diagonal targets were considered. Near transfer was examined in two ways: Response times to targets at the start of training in $\mathrm{C}$ were compared with those to new targets at the start of testing in D1 and D2, and response times to targets at the start of testing in $\mathrm{C}$ were compared with those to new targets at the start of testing in D1 and D2. If response times to new targets at the start of testing in the experimental conditions were found to be shorter than those to targets at the start of training in the control condition by the first analysis, there would be some evidence for transfer of training. However, such transfer might only be partial and not full. If response times to new targets at the start of testing in the experimental conditions were found to be equal to those to targets at the start of testing in the control condition (in which those targets had been practiced during training) by the second analysis, only then would there be evidence for full transfer.

First, an ANOVA was conducted in which we compared response times on new diagonal targets in the first five blocks of testing in D1 and D2 with response times in the first five blocks of training in $\mathrm{C}$ (for all diagonal targets). There was a main effect of condition, with longer MTs during training in the $\mathrm{C}$ condition $(M=2.127 \mathrm{sec})$ than during testing in the D1 $(M=1.418 \mathrm{sec})$ and the D2 $(M=1.057 \mathrm{sec})$ conditions $\left[F(2,63)=33.52, M S_{\mathrm{e}}=\right.$ 

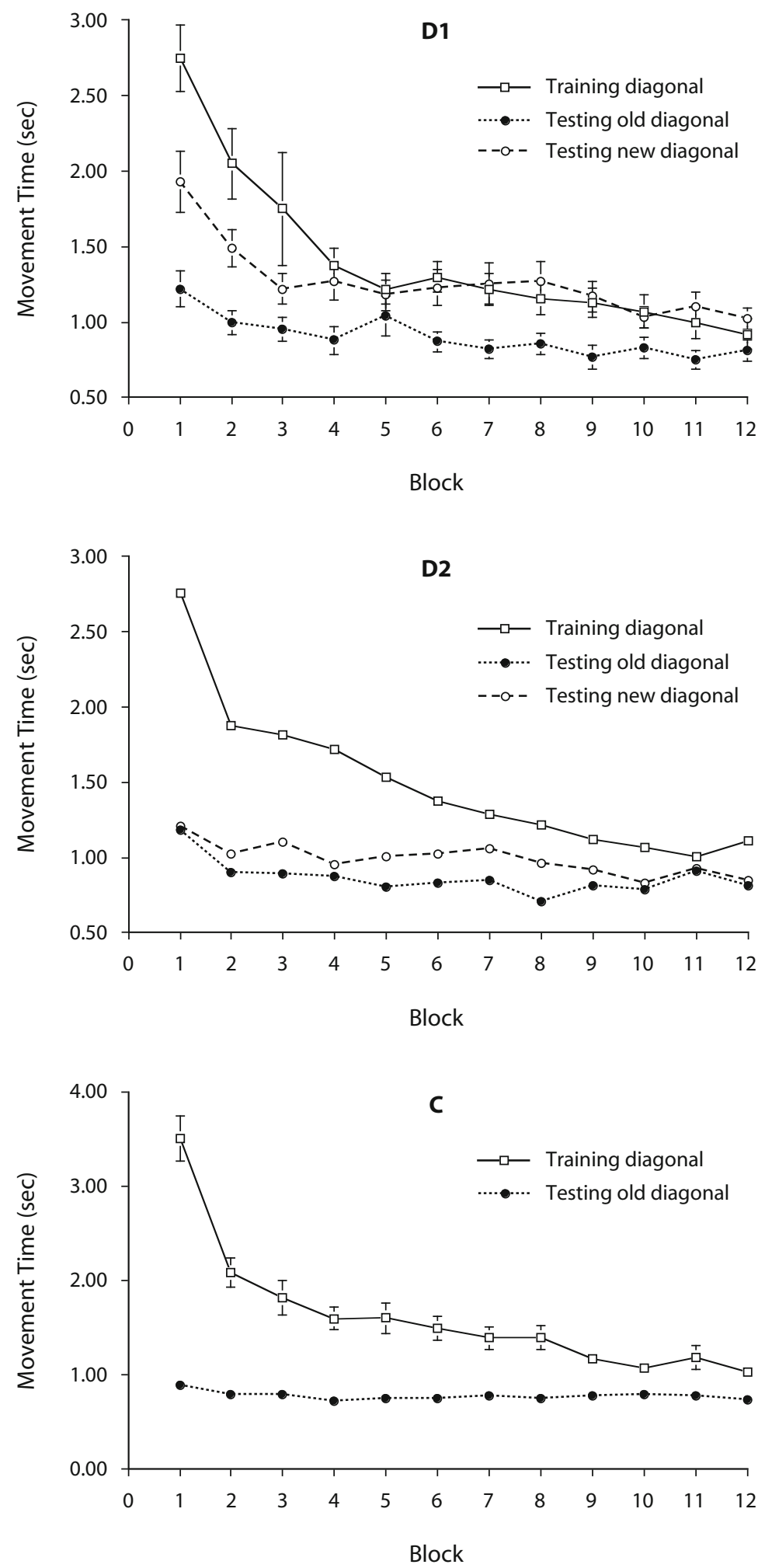

Figure 2. Mean movement times for diagonal targets during training and testing for the D1 condition (top panel), the D2 condition (middle panel), and the $\mathbf{C}$ condition (bottom panel) as a function of block and target type. Error bars represent standard errors of the means. 
$0.972, p<.01]$. A planned comparison of MTs at test in the D1 and D2 conditions revealed a main effect of condition $\left[F(1,42)=9.46, M S_{\mathrm{e}}=0.758, p<.01\right]$, with shorter MTs for participants in the D2 condition than in the D1 condition. Additional planned tests were conducted, comparing each experimental condition (D1 and D2) separately with the control condition (C). Both the analysis involving D1 $\left[F(1,42)=21.62, M S_{\mathrm{e}}=1.277, p<.01\right]$ and the analysis involving D2 $\left[F(1,42)=71.54, M S_{\mathrm{e}}=\right.$ $0.880, p<.01]$ yielded a significant effect of condition, documenting significant partial transfer in each case. In the overall analysis, there was also a main effect of block $\left[F(4,252)=39.70, M S_{\mathrm{e}}=0.264, p<.01\right]$ and an interaction of block and condition $\left[F(8,252)=11.49, M S_{\mathrm{e}}=\right.$ $0.264, p<.01$ ], reflecting greater improvement across blocks in the $\mathrm{C}$ condition (during training) than in the $\mathrm{D} 1$ and $\mathrm{D} 2$ conditions (during test).

Second, an ANOVA compared response times on the first five blocks of testing in the three conditions. The main effect of condition was significant $[F(2,63)=5.39$, $\left.M S_{\mathrm{e}}=0.947, p<.01\right]$, with longer response times for participants in the D1 condition $(M=1.418 \mathrm{sec})$ than for those in the $\mathrm{D} 2(M=1.057 \mathrm{sec})$ or the $\mathrm{C}(M=1.034 \mathrm{sec})$ condition. Planned tests were conducted, comparing each experimental condition (D1 and D2) separately with the control condition (C). The analysis involving D1 did reveal a significant difference $\left[F(1,42)=6.55, M S_{\mathrm{e}}=\right.$ $1.240, p=.01]$, whereas the analysis involving $\mathrm{D} 2 \mathrm{did}$ not $[F(1,42)<1]$, suggesting that transfer was full for the D2 condition but not for the D1 condition. Again, the main effect of block was also significant $[F(4,252)=$ $\left.17.19, M S_{\mathrm{e}}=0.141, p<.01\right]$, with improvement across blocks, and the improvement was larger for participants in the $\mathrm{D} 1$ condition than for those in either the $\mathrm{C}$ or the $\mathrm{D} 2$ condition $\left[F(8,252)=2.24, M S_{\mathrm{e}}=0.141, p=.03\right]$ (see Figure 2).

Far transfer. For the assessment of far transfer, only targets along the major axes (horizontal and vertical) were considered, rather than diagonal targets. Far transfer was examined in the same two ways as was near transfer.

First, an ANOVA was conducted on targets along the major axes, in which we compared response times in the first five blocks of testing in D1 and D2 with response times in the first five blocks of training in $\mathrm{C}$. The factor of axis (vertical, horizontal) was added to the analysis. There was a main effect of condition, with longer MTs during training in the $\mathrm{C}$ condition $(M=1.560 \mathrm{sec})$ than during testing in the $\mathrm{D} 1(M=0.963 \mathrm{sec})$ and the $\mathrm{D} 2(M=$ $0.769 \mathrm{sec})$ conditions $\left[F(2,63)=27.85, M S_{\mathrm{e}}=1.342\right.$, $p<.01]$. A planned comparison of MTs at test in the D1 and D2 conditions revealed a main effect of condition $\left[F(1,42)=5.95, M S_{\mathrm{e}}=0.696, p=.02\right]$, with shorter MTs for participants in the D2 condition than in the D1 condition. Additional planned tests were conducted, comparing each experimental condition (D1 and D2) separately with the control condition (C). Both the analysis involving D1 $\left[F(1,42)=21.18, M S_{\mathrm{e}}=1.851, p<.01\right]$ and the analysis involving D2 $\left[F(1,42)=46.49, M S_{\mathrm{e}}=1.480, p<\right.$ $.01]$ yielded a significant effect of condition, documenting significant partial transfer in each case. In the overall analysis, there was also a main effect of block $[F(4,252)=$ $\left.11.71, M S_{\mathrm{e}}=0.428, p<.01\right]$ and an interaction of block and condition $\left[F(8,252)=4.71, M S_{\mathrm{e}}=0.428, p<.01\right]$, reflecting much greater improvement across blocks in the $\mathrm{C}$ condition (during training) than in the D1 and D2 conditions (during test; see Figure 3). In addition, there was a main effect of axis, with shorter response times to targets along the (reversed) horizontal axis $(M=0.822 \mathrm{sec})$ than along the (nonreversed) vertical axis $(M=1.372 \mathrm{sec})$ $\left[F(1,63)=84.49, M S_{\mathrm{e}}=0.592, p<.01\right]$. There was also a significant interaction between condition and axis $\left[F(2,63)=11.58, M S_{\mathrm{e}}=0.592, p<.01\right]$. The difference between the two axes was larger in the $\mathrm{C}$ condition (during training) than in the D1 and D2 conditions (during test) (C horizontal, $M=1.084 \mathrm{sec}$; $\mathrm{C}$ vertical, $M=$ $2.036 \mathrm{sec}$; D1 horizontal, $M=0.759 \mathrm{sec}$; D1 vertical, $M=1.167 \mathrm{sec}$; D2 horizontal, $M=0.624 \mathrm{sec}$; D2 vertical, $M=0.914 \mathrm{sec}$ ).

Second, an analysis compared response times in the three conditions during the first five blocks of testing. The main effect of condition was significant $[F(2,63)=$ $\left.7.34, M S_{\mathrm{e}}=0.563, p<.01\right]$, with longer response times for participants in the $\mathrm{D} 1$ condition $(M=0.963 \mathrm{sec})$, than for those in either the D2 $(M=0.769 \mathrm{sec})$ or the $\mathrm{C}(M=$ $0.698 \mathrm{sec}$ ) condition. Planned tests were conducted, comparing each experimental condition (D1 and D2) separately with the control condition $(\mathrm{C})$. The analysis involving D1 yielded a significant difference $[F(1,42)=11.30$, $\left.M S_{\mathrm{e}}=0.683, p<.01\right]$, but that involving D2 did not $\left[F(1,42)=1.78, M S_{\mathrm{e}}=0.312, p=.19\right]$, suggesting that transfer was full for D2 but only partial for D1. Again, the main effect of block was significant $[F(4,252)=7.80$, $\left.M S_{\mathrm{e}}=0.086, p<.01\right]$, with improvement across blocks (see Figure 3). The main effect of axis was also significant $\left[F(1,63)=106.48, M S_{\mathrm{e}}=0.186, p<.01\right]$, reflecting shorter response times for targets along the (reversed) horizontal axis $(M=0.637 \mathrm{sec})$ than for those along the (nonreversed) vertical axis $(M=0.983 \mathrm{sec})$.

\section{Initiation Times}

Training. The participants in the three conditions improved in their IT as a function of practice $[F(11,693)=$ $\left.16.91, M S_{\mathrm{e}}=0.024, p<.01\right]$. There was also a significant main effect of condition $\left[F(2,63)=15.84, M S_{\mathrm{e}}=0.303\right.$, $p<.01]$. As was expected on the basis of the Hick-Hyman law (e.g., Hick, 1952), ITs were longer in the C condition, which had all eight targets $(M=0.896 \mathrm{sec})$, than in the D1 $(M=0.639 \mathrm{sec})$ and D2 $(M=0.695 \mathrm{sec})$ conditions, which had only two targets. There was also a significant interaction of block and condition $[F(22,693)=$ $\left.2.10, M S_{\mathrm{e}}=0.024, p<.01\right]$, apparently reflecting less improvement across blocks in the D1 condition than in the $\mathrm{D} 2$ or $\mathrm{C}$ conditions. In an analysis restricted to the D1 and D2 conditions, no effects of condition were found.

Retention. As for MT, to examine retention, we compared IT on the last five blocks of training in the $\mathrm{C}$ condition to that on the first five blocks of testing in the D1 and D2 conditions for old targets. The main effect of condition was not significant $\left[F(2,63)=1.52, M S_{\mathrm{e}}=0.347, p=\right.$ .23], reflecting essentially perfect retention for the two 

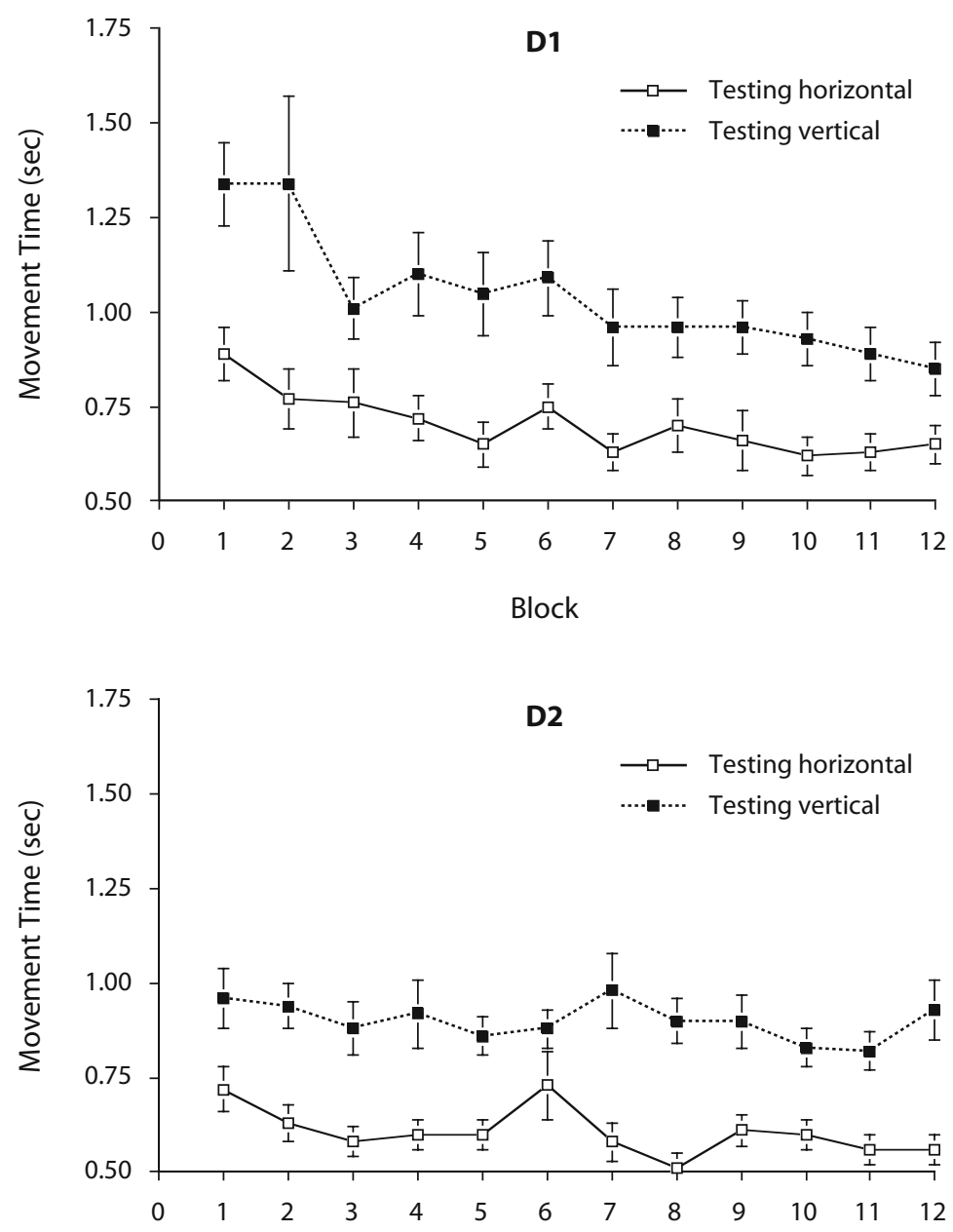

Block

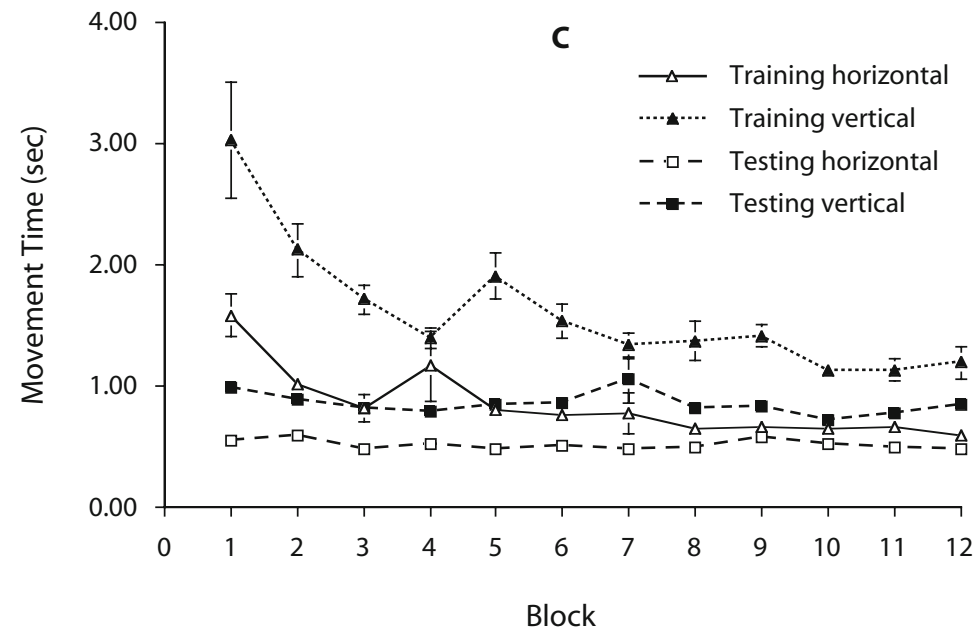

Figure 3. Mean movement times for targets along the major axes during training (in the $C$ condition) and testing (in all conditions) for the $D 1$ condition (top panel), the D2 condition (middle panel), and the $C$ condition (bottom panel) as a function of block and target type. Error bars represent standard errors of the means. 
experimental conditions by this measure, as was found for MT (D1, $M=0.937 \mathrm{sec}$; D2, $M=0.876 \mathrm{sec}$; C, $M=$ $0.799 \mathrm{sec})$. However, ITs did improve across blocks $\left[F(4,252)=8.07, M S_{\mathrm{e}}=0.046, p<.01\right]$.

Specificity. As for MT, there was an advantage for old $(M=0.782 \mathrm{sec})$ over new $(M=0.856 \mathrm{sec})$ target digits $\left[F(1,42)=9.94, M S_{\mathrm{e}}=0.147, p<.01\right]$, demonstrating specificity of training. In addition, ITs decreased across blocks of testing $\left[F(11,462)=12.19, M S_{\mathrm{e}}=0.041, p<\right.$ $.01]$ (see Figure 4 ). The interaction of target type and block was not significant, and no effects involving condition were significant.

Near transfer. As was done for MT, an initial analysis was conducted for IT to determine whether there was any partial transfer. ITs for new targets at the start of testing in the D1 $(M=0.954 \mathrm{sec})$ and D2 $(M=0.890 \mathrm{sec})$ conditions did not differ statistically from those at the start of training in the $\mathrm{C}(M=0.968 \mathrm{sec})$ condition $[F(2,63)<1]$. Separate planned comparisons between each experimental condition and the control condition also revealed no effects of condition, documenting no significant partial transfer. All conditions improved across blocks (see Figure 4) $\left[F(4,252)=8.42, M S_{\mathrm{e}}=0.054, p<.01\right]$. Because this analysis yielded no evidence for partial transfer, no examination of the possibility of full transfer was undertaken.

Far transfer. For the assessment of far transfer, only targets along the major axes (horizontal and vertical) were considered, rather than diagonal targets. As with the assessment of near transfer, only partial transfer was examined. ITs for new targets at the start of testing in the D1 $(M=0.863 \mathrm{sec})$ and $\mathrm{D} 2(M=0.878 \mathrm{sec})$ conditions did not differ statistically from those at the start of training in the $\mathrm{C}(M=0.899 \mathrm{sec})$ condition $[F(2,63)<1]$, suggesting no evidence for even partial far transfer. There was a main effect of block $\left[F(4,252)=25.83, M S_{\mathrm{e}}=0.033, p<.01\right]$, reflecting improvement with practice, and a main effect of axis $\left[F(1,63)=57.81, M S_{\mathrm{e}}=0.060, p<.01\right]$, reflecting, again, shorter ITs along the (reversed) horizontal axis $(M=0.808 \mathrm{sec})$ than along the (nonreversed) vertical axis $(M=0.952 \mathrm{sec})$. In addition, there was an interaction of block and axis $\left[F(4,252)=3.74, M S_{\mathrm{e}}=0.027, p<.01\right]$, reflecting more improvement across blocks along the horizontal axis than along the vertical axis (see Figure 5).

\section{DISCUSSION}

The results for MT and for IT yield different patterns, suggesting that the various components of the speeded aiming task show different amounts of retention and transfer. Although future research should include kinematic measures for trajectory and velocity data to allow for a more comprehensive assessment of transfer, several important conclusions can still be drawn from the present response time data.

\section{Movement Time}

Although MTs were quite long at the start of training, presumably because learning the mouse reversal was required, the participants showed improvement during train- ing and perfect retention, with no loss in performance (in fact, with some improvement) between the end of training and beginning of testing for old targets. Evidence of specificity of training was found because, at testing, responses to old targets were faster than those to new targets, and the advantage for old targets did not decrease across testing blocks. This difference between old and new targets was found in both conditions, although it was significantly larger in the D1 than in the D2 condition. Finding that MTs for old targets were shorter than those for new targets in the $\mathrm{D} 2$ condition is especially interesting because all diagonal movements were trained in the D2 condition, if return as well as instructed movements are considered. Thus, the present results suggest that instructed movements are learned to a greater extent than are return movements. Because instructed and return movements are equivalent with respect to the direction and extent of movement, these results also imply that learning is specific to the cursor endpoints (i.e., the starting and goal locations), as well as to the direction and extent of movement.

There are at least two complementary additional explanations for the finding of faster responses at test to old than to new targets in the D2 condition. The first concerns not the differing cursor endpoints for instructed and return movements but, rather, the different directions of the mouse with respect to the body. The return and instructed mouse movements always differ in terms of whether they are toward the body (pull) or away from the body (push). It has been shown that there are in fact behavioral differences between pulling and pushing, supporting the view of embodied cognition (e.g., Markman \& Brendl, 2005). The second additional explanation for this finding concerns effects of intention on learning, assuming that the instructed movements are learned intentionally, whereas the return movements are learned incidentally. Intentional learning has been shown under some circumstances to be more effective than incidental learning (e.g., Eagle \& Leiter, 1964; Marmie \& Healy, 2004). Viewed in this way, the present findings extend previous demonstrations of the advantages of intentional learning from verbal and perceptual learning to motor learning.

The analysis comparing performance at the beginning of training in the control condition (C) to performance at the beginning of testing for new targets in the D1 and D2 experimental conditions provided evidence for partial transfer for both experimental conditions, although transfer was larger for the condition involving both diagonals (D2) than for the condition involving a single diagonal (D1). For the D2 condition (but not for the D1 condition), there was also evidence for full transfer of learning, because performance at the beginning of testing was not significantly different from that at the beginning of testing in the $\mathrm{C}$ condition (in which the participants had been trained with all targets). These findings concerning transfer held for both near transfer to new diagonal targets and far transfer to new targets on one of the major axes.

In summary, for MT, there were differences observed between the D1 and the D2 condition in the amount of specificity and transfer. Specifically, less specificity (i.e., a smaller difference between old and new targets at test) 

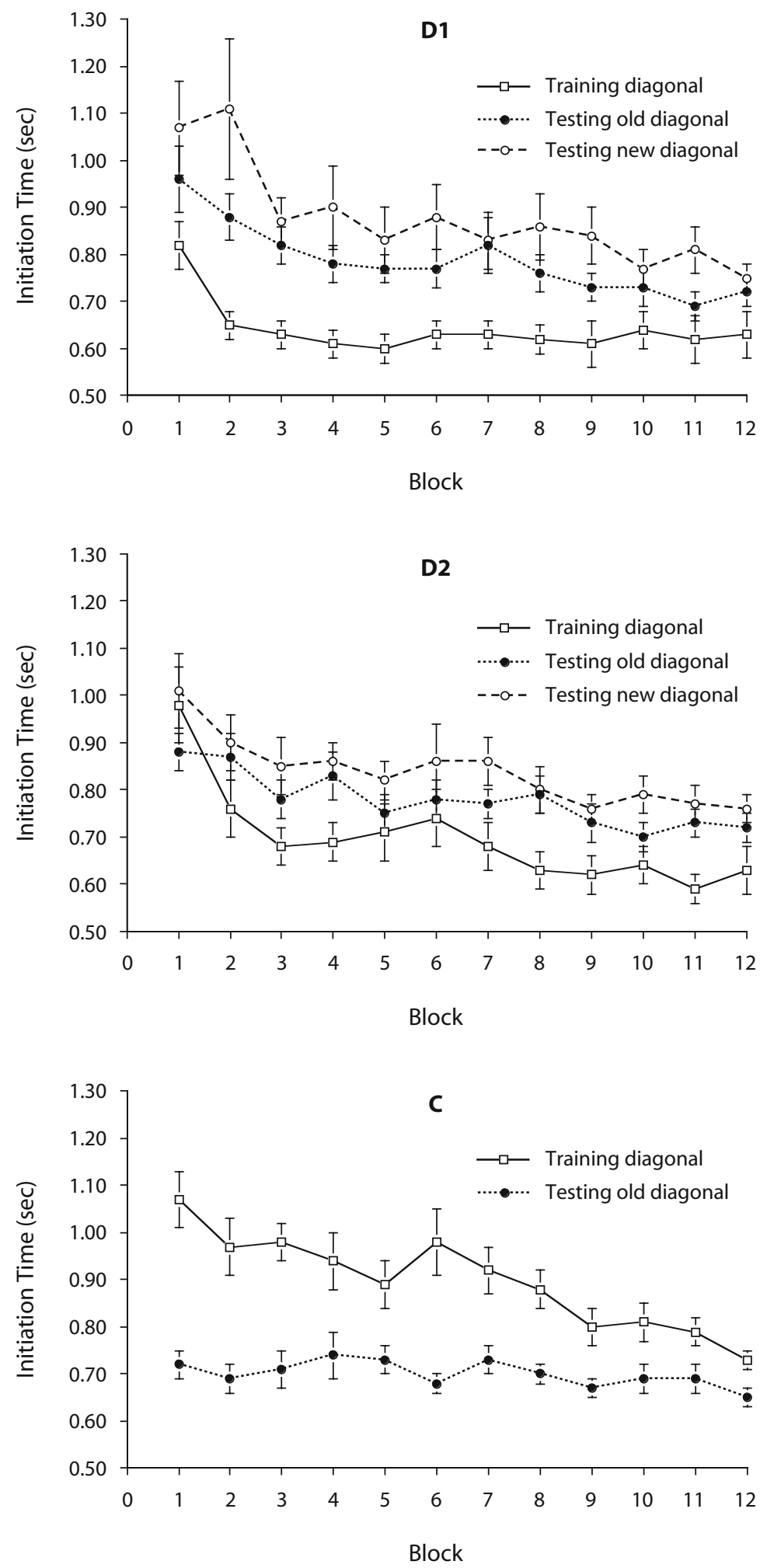

Figure 4. Mean initiation times for diagonal targets during training and testing for the D1 condition (top panel), the D2 condition (middle panel), and the $\mathrm{C}$ condition (bottom panel) as a function of block and target type. Error bars represent standard errors of the means. 

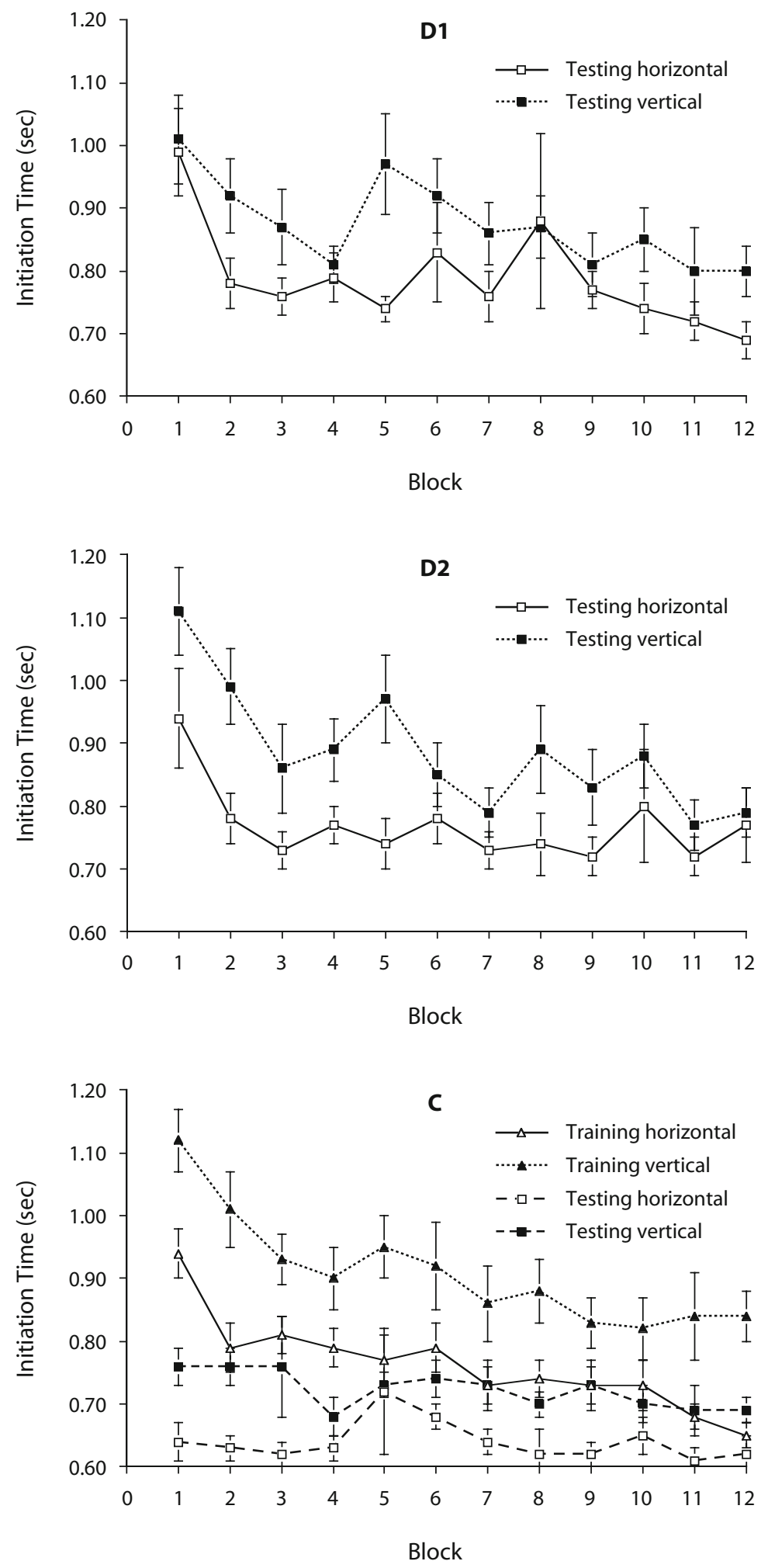

Figure 5. Mean initiation times for targets along the major axes during training (in the $\mathrm{C}$ condition) and testing (in all conditions) for the D1 condition (top panel), the D2 condition (middle panel), and the $C$ condition (bottom panel) as a function of block and target type. Error bars represent standard errors of the means. 
and more transfer were found for D2, which involved training on both diagonals, than for D1, which involved training on a single diagonal. Nevertheless, partial transfer of MTs (comparing the beginning of training with the beginning of testing) was evident for both conditions. These findings can be explained if participants learn not only how to go from the center starting point to the target (the instructed movements) but also how to return from the target to the center starting point. By that explanation, the participants in the D2 condition learned during training how to make movements in all four directions (northeast, southeast, northwest, and southwest), whereas the participants in the D1 condition learned during training how to make movements in only two of the four directions (northeast and southwest or southeast and northwest). Thus, the return movements back to the start position, not just the instructed movements to the target, must contribute to learning during training to some extent, although as was mentioned earlier, return movements are not learned to the same extent as instructed movements.

\section{Initiation Time}

Like MT, IT improved with training and showed perfect retention. As was found for MT, we found additional support for specificity of learning for IT, because, at test, responses to old targets were faster than those to new targets, and the advantage for old targets did not decrease across testing blocks. However, there was no evidence of either near or far partial transfer for IT, because performance on new targets at the start of testing in the experimental conditions (D1 and D2) was not better than performance at the start of training in the control condition (C). On the assumption that IT reflects largely encoding and planning of movements, but MT reflects largely execution of movements (see Bourne et al., 2005; Elliott \& Lee, 1995; Khan et al., 2006; Rosenbaum, 1980), this finding, taken together with the results of full transfer for MT, suggests that there is more transfer of learned movement execution than of encoding or planning those movements.

Despite the large differences between MT and IT in the pattern of results for the transfer analyses (revealing not even partial transfer for IT but full transfer in one condition and partial transfer in another condition for MT), the two measures agree in showing specificity of training. In both cases, old targets were responded to more rapidly than new targets at test. Finding a significant effect in the specificity analysis of IT suggests that the lack of a transfer effect for IT is unlikely to be due to a lack of sensitivity of that measure.

\section{Variability of Practice}

The variability-of-practice hypothesis led to the prediction of more transfer for participants in the $\mathrm{D} 2$ condition (who practiced movements in all four diagonal directions, including both instructed and return movements) than for those in the D1 condition (who practiced movements in only two diagonal directions, including both instructed and return movements). Indeed, more transfer of MT was found for D2 than for D1 in terms of both near and far transfer. Because there was equal variability in D1 and D2 with respect to the instructed movements during training (two instructed movements in each case), finding more transfer for D2 than for D1 implies that variability of practice applies to return movements, as well as to instructed movements. Moreover, because the results for far transfer yielded the same pattern as did those for near transfer, the findings imply that the advantage for variable practice applies not only to similar movements but also to more dissimilar movements that were not practiced in any way (i.e., neither as instructed nor as return movements).

\section{Global Inhibition}

On the basis of their findings concerning speeded aiming, Healy et al. (2006) proposed a global inhibition hypothesis, according to which participants applied a global inhibition strategy to cope with the mouse reversal encountered in this task. By this strategy, all normal mouse movements are inhibited (i.e., suppressed), but when only horizontal, not vertical, movements are reversed, participants must take the added step of disinhibiting responses along the vertical dimension. This extra step increases MT to those targets located along the vertical axis, leading to the prediction of longer times for pure vertical movement than for pure horizontal movement, which, although unintuitive, was indeed found by Healy et al. (2006) and was replicated here in the assessment of far transfer. In the present study, if the participants did adopt a global inhibition strategy, there should have been positive transfer to the untrained targets along both of the diagonal axes, as well as along the horizontal and vertical major axes, because both trained and untrained targets on these axes require that horizontal movements be inhibited and vertical movements be disinhibited in the same way. That is, according to the global inhibition hypothesis alone, no differences in transfer should have been found for trained and untrained movements or for the D1 and D2 conditions. The present results, along with the earlier results of Wohldmann et al. (2008), are, thus, not consistent with the global inhibition hypothesis on its own and require a provision for movement tactics that are specific to each target. Because some transfer of return movements was found (i.e., the responses in the D2 condition were faster than those in the D1 condition on new targets at test), these findings also suggest that the specific movement tactics for targets along the same diagonal overlap or are highly similar. However, because the participants in the D2 condition, who practiced all diagonal movements at training as either instructed or return movements, showed shorter MTs at test for the movements that had been instructed at training than for those that had been only return movements at training, the specific movement tactics for the instructed and return movements must differ to some extent. Also, because transfer was substantial to the targets along the horizontal and vertical axes, which had not been practiced in the D1 and D2 conditions, the participants must have learned more than just specific movement tactics applicable to diagonal targets. That is, the participants seem to have learned both the global inhibition strategy and particular movements that depended on their starting and goal locations. 


\section{Conclusions}

Taken together, the findings from this experiment support the advantages of variable practice and demonstrate that specificity and transfer of learning are not mutually exclusive. Significant specificity can be found even when participants fully transfer what they have learned to performance at test. Furthermore, depending on how transfer is defined (e.g., near or far, partial or full) and what the measure of performance is (e.g., MT or IT), different amounts of transfer might be evident. Specifically, although the same pattern of results was found for near and far transfer, the results for the measure MT demonstrated full transfer in the D2 condition but only partial transfer in the D1 condition, and there was no evidence of even partial transfer for the measure IT. These are especially important findings, given that in many experiments in cognitive psychology, total response time is used as the dependent variable, rather than multiple component response times, although multiple measures are more common in motor control research. Thus, the use of different definitions of transfer and a single crude measure in previous investigations might explain why there has been little consensus about the conditions under which durability, specificity, and transfer of learning occur.

\section{AUTHOR NOTE}

This research was supported in part by Army Research Office Grant W911NF-05-1-0153 to the University of Colorado. We thank Ani Gharibyan and Sasoon Arutyunyan for help testing participants and Lyle Bourne, Jay Holden, and Martina Rieger for valuable advice about this research. A summary of a preliminary version of this study was presented at the 2008 meeting of the Psychonomic Society in Chicago. Correspondence concerning this article should be addressed to E. L. Wohldmann, Department of Psychology, California State University, Northridge, 18111 Nordhoff St., Northridge, CA 91330-8255 (e-mail: erica.wohldmann@csun.edu).

\section{REFERENCES}

Bourne, L. E., Jr., Healy, A. F., Pauli, P., Parker, J. T., \& BirBAUMER, N. (2005). The influence of stimulus array on training of a speeded response. American Journal of Psychology, 118, 385-411.

Eagle, M., \& Leiter, E. (1964). Recall and recognition in intentional and incidental learning. Journal of Experimental Psychology, 68, 58-63. doi: $10.1037 / \mathrm{h} 0044655$

Elliott, D., \& LeE, T. D. (1995). The role of target information on manual-aiming bias. Psychological Research, 58, 2-9.

Healy, A. F., Wohldmann, E. L., \& Bourne, L. E., JR. (2005). The procedural reinstatement principle: Studies on training, retention, and transfer. In A. F. Healy (Ed.), Experimental cognitive psychology and its applications (pp. 59-71). Washington, DC: American Psychological Association.

Healy, A. F., Wohldmann, E. L., Sutton, E. M., \& Bourne, L. E., JR. (2006). Specificity effects in training and transfer of speeded responses. Journal of Experimental Psychology: Learning, Memory, \& Cognition, 32, 534-546. doi:10.1037/0278-7393.32.3.534

Hick, W. E. (1952). On the rate of gain of information. Quarterly Journal of Experimental Psychology, 4, 11-26.

Khan, M. A., Lawrence, G. P., Buckolz, E., \& Franks, I. M. (2006). Programming strategies for rapid aiming movements under simple and choice reaction time conditions. Quarterly Journal of Experimental Psychology, 59, 524-542.

Markman, A. B., \& Brendl, C. M. (2005). Constraining theories of embodied cognition. Psychological Science, 16, 6-10. doi:10.1111/ j.0956-7976.2005.00772.x

Marmie, W. R., \& Healy, A. F. (2004). Memory for common objects: Brief intentional study is sufficient to overcome poor recall of US coin features. Applied Cognitive Psychology, 18, 445-453. doi:10.1002/ acp.994

Morris, C. D., Bransford, J. D., \& Franks, J. J. (1977). Levels of processing versus transfer appropriate processing. Journal of Verbal Learning \& Verbal Behavior, 16, 519-533. doi:10.1016/S0022 $-5371(77) 80016-9$

Proteau, L., Marteniuk, R. G., \& Lévesque, L. (1992). A sensorimotor basis for motor learning: Evidence indicating specificity of practice. Quarterly Journal of Experimental Psychology, 44A, 557-575.

Roediger, H. L., III, Weldon, M. S., \& Challis, B. H. (1989). Explaining dissociations between implicit and explicit measures of retention: A processing account. In H. L. Roediger III, \& F. I. M. Craik (Eds.), Varieties of memory and consciousness: Essays in honour of Endel Tulving (pp. 3-41). Hillsdale, NJ: Erlbaum.

Rosenbaum, D. A. (1980). Human movement initiation: Specification of arm, direction, and extent. Journal of Experimental Psychology: General, 109, 444-474. doi:10.1037/0096-3445.109.4.444

Schmid, R. A. (1975). A schema theory of discrete motor skill learning. Psychological Review, 82, 225-260. doi:10.1037/h0076770

SchmidT, R. A., \& BJorK, R. A. (1992). New conceptualizations of practice: Common principles in three paradigms suggest new concepts for training. Psychological Science, 3, 207-217. doi:10.1111/ j.1467-9280.1992.tb00029.X

Tulving, E., \& Thomson, D. M. (1973). Encoding specificity and retrieval processes in episodic memory. Psychological Review, 80, 352373. doi: $10.1037 / \mathrm{h} 0020071$

Wohldmann, E. L., Healy, A. F., \& Bourne, L. E., JR. (2008). Global inhibition and midcourse corrections in speeded aiming. Memory \& Cognition, 36, 1228-1235. doi:10.3758/MC.36.7.1228

WuLF, G., \& SCHMIDT, R. A. (1997). Variability of practice and implicit motor learning. Journal of Experimental Psychology: Learning, Memory, \& Cognition, 23, 987-1006. doi:10.1037/0278-7393.23.4.987

(Manuscript received July 20, 2009; revision accepted for publication October 7, 2009.) 